\title{
Quantitative radiomic phenotyping of cervix cancer
}

\begin{abstract}
Radiomics aims to extract huge amount of quantifiable features from medical images with data-characterization algorithms.1-5 Radiologists cannot appreciate all the disease characteristics in generic diagnostic images.6 Using these radiomic features, it is possible to uncover the physiognomies of disease progression. The hypothesis of radiomics is that the distinctive imaging features between disease forms may be useful for predicting prognosis and therapeutic response for various conditions, thus providing valuable information for personalized therapy.1,7,8 Radiomics emerged from oncology 3 is the most cutting-edge innovation. However, the technique can be useful in any medical study involving tomographic imaging. This article analyzes currently available phenotyping algorithms for Lung and Head \& Neck malignancies. And It can help researchers to understand the concept to apply the same for cervix cancer.
\end{abstract}

Keywords: radiomics, feature selection, cervix cancer
Volume 6 Issue I - 2018

\author{
Surega Anbumani,' Punitha Jayaraman,' \\ Pichandi Anchaneyan,' Ramesh S Bilimagga,' \\ Arunai Nambiraj $\mathrm{N}^{2}$ \\ 'HCG Bangalore Institute of Oncology, India \\ ${ }^{2}$ Centre for Biomaterials, Cellular and Molecular Theranostics, \\ VIT University, India
}

Correspondence: Surega Anbumani, HCG Bangalore Institute of Oncology, Bangalore, India, Email suregaanbumani@gmail.com

Received: December 30, 2017 | Published: January 30, 2018

\section{Introduction}

Radiomics process comprises of following five major levels.

i. Image acquisition (Computed Tomography imaging)

ii. Developing algorithms for image segmentation (Automatic/Semi automatic).

iii. Extraction and feature quantification

iv. Analysis and creation of database

v. Developing retrospective/prognostic models for personalized therapy.

Large volumes of raw scan images are stored in DICOM RT format in Picture Archiving and Communication System in imaging department. Thus, it is made available to data mining procedures. Raw data does not contain any tumor volumes defined. Hence the transverse $\mathrm{CT}$ images are segmented slice by slice using any automatic or semiautomatic algorithms. Many radiomic features are computed from the images after segmentation. The features range from volume, shape, surface density and intensity, texture, tumor location, relations with the surrounding tissues. Redundant information can be eliminated by proper quantification of data acquired. A feature selection algorithm accelerates the evaluation of these features. The data in the radiomic features were compared for any similarity. The repetition of similarity is evaluated when occurs in the same time frame. An outcome variable is defined in supervised analysis of data points to create a predictive model. Graphical representation of final results is deduced from unsupervised analysis. Now the new patient CT data is given as inputs in an radiomic algorithm, that returns a value for tumor growth and disease free survival. Radiomic features can be grouped into five important characteristics such as;

i. Size and shape based-features, image intensity histogram descriptors,

ii. Image-voxels descriptors (e.g. Gray-level co-occurrence matrix $(\mathrm{glcm})$, run length matrix $(\mathrm{rlm})$, size zone matrix (szm), iii. And neighborhood gray tone difference matrix (ngtdm) derived textures,

iv. Filtered images textures, and

v. Fractal features.

The mathematical definitions (algorithms) of these radiomic features are independent of imaging modality (suitable for ultrasound, MRI imaging etc.). ${ }^{10-14} \mathrm{~A}$ detailed description of texture features for radiomics can be found in Depeursinge et al. ${ }^{15}$

\section{Methods}

A retrospective data collection of $\mathrm{CT}$ images of 22patients treated in the clinic from the year 2015 to 2017. Our article outlines the design of radiomics study of cervix cancer. Patients were included based on specific selection criteria as mentioned as follows;

\section{Inclusion criteria}

i. Histopathological confirmed locally advanced Squamous intra epithelial cell carcinomas of Cervix

ii. Age $<65$ years

iii. ECOG performance status of $0-2$.

iv. Hematological parameters with total leukocyte count of $>4000$ cells $/ \mathrm{mm}^{3}$,

v. platelet counts of $>1.51 \mathrm{akh} / \mathrm{mm}^{3}$

vi. Renal parameters with Serum creatinine

vii. Patients with an informed consent.

\section{Exclusion criteria}

i. Tumors of non - squamous histology.

ii. Age $>65$ years.

iii. Performance status ECOG PS $>2$.

iv. Any prior treatment received for the tumor. 
v. Patients with abnormal cardiac function, renal, hematological parameters or co-morbid illness.

vi. Patients who do not give an informed consent.

vii. Patients unavailable for follow up

CT images archived from PACS were contoured with normal structures such as Rectum, Urinary Bladder, Intestines, Left and right femoral bones using an automatic edge detection algorithm using 3D slicer software in an independent system (Figure 1). And the Gross tumor volume, Clinical Target Volume, high risk/low risk tumor volumes were delineated with semi automatic algorithm under the supervision of experienced radiation oncologists. A Combined (MATLAB and $\mathrm{C}++$ ) code was generated using grey scale differentiations and contoured volumes to extract the features. These features can be used as predictive models for outcome prediction, distant metastasis risk analysis and genetics assessment, when a new patient data was given as input (Figure 2).

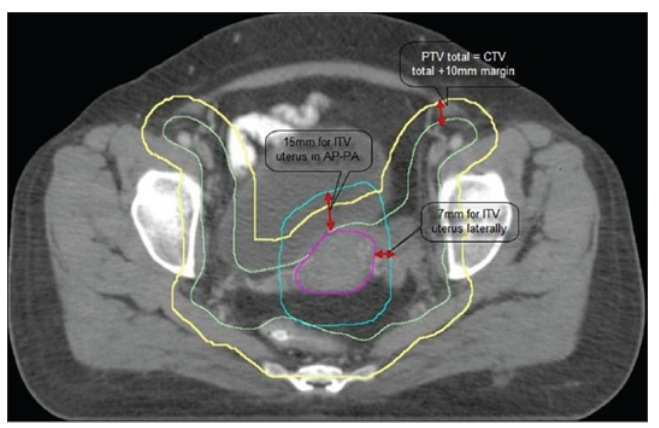

Figure I Gross tumor volume.

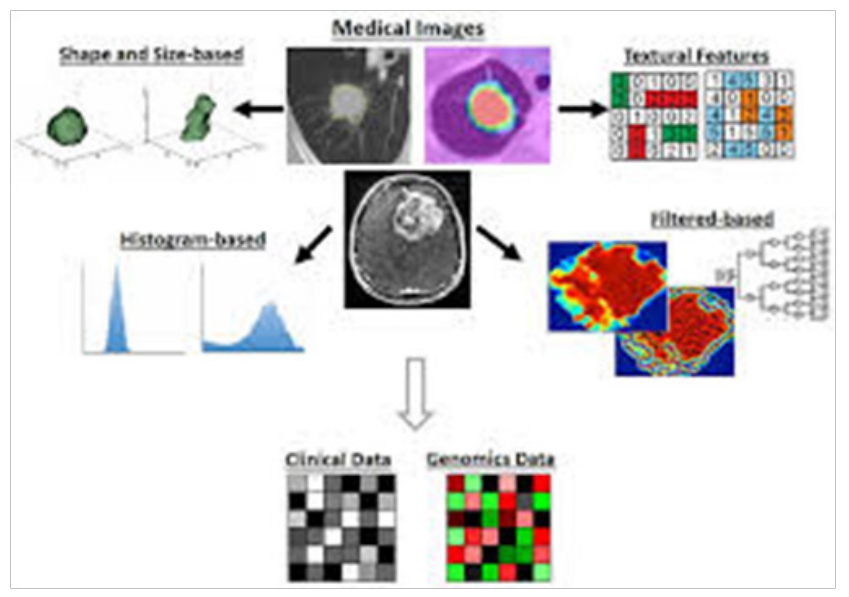

Figure 2 Patient data was given.

\section{Discussion}

Clinical outcome prediction was first performed by Aerts et al. It was a large-scale radiomic study that included three lung and two head-and-neck cancer cohorts with 1000patients. They evaluated the predictive values of more than 400 textural and shape- and intensitybased features extracted from the computed tomography (CT) images acquired before treatment. Tumor volumes were delineated either by radiation oncologists or using semiautomatic segmentation methods. $^{16,17}$ A subset of radiomic features were identified for predicting patient survival and describing intra-tumoral heterogeneity.
It was confirmed that the prognostic ability of these radiomics features may also be used for head-and-neck cancer. But later it was found that prognostic value of some radiomic features may be cancer type dependent. ${ }^{18}$ Not every radiomic feature that significantly predicted the survival of lung cancer patients could also predict the survival of head-and-neck cancer patients and vice versa. Radiomic features are better at predicting treatment response than conventional measures, such as tumor volume and diameter, and the maximum radiotracer uptake on positron emission tomography (PET) imaging. ${ }^{19-25}$ Metastatic latency of tumors could also be predicted by radiomic features. ${ }^{26-27}$ For example, 35 CT-based radiomic features were recognized to be predictive of distant metastasis of lung cancer ${ }^{26}$ Thus it guides physicians to select the effective treatment for individual patients with a risk of developing distant metastasis. Cancer genetics Assessment associated with biological gene sets, such as cell cycle phase, DNA recombination, immune system regulatory process. ${ }^{1}$ Moreover, various mutations of glioblastoma (GBM), such as $1 \mathrm{p} / 19 \mathrm{q}$ deletion, MGMT methylation, TP53, EGFR, and NF1, were predicted by magnetic resonance imaging (MRI) volumetric measures, including tumor volume, necrosis volume, and contrast enhancing volume. ${ }^{28-30}$ Radiomic feature extracted with FDG PET scan of cervix cancer deals with the Texture analysis of tumor. ${ }^{15,29,30}$ But, in our study we have extracted tumor signatures using computed tomography images.

\section{Conclusion}

We developed an empirical algorithm for feature extraction with $\mathrm{CT}$ data sets of 22 cervix cancer patients. A preliminary development of algorithm was done in a stand-alone computer system with MATLAB and $\mathrm{C}++$ environment. Radiomic features extracted to define squamous cell carcinoma of cervix phenotype were: Textural feature, Tumor size and heterogeneity. Outcome and metastatic risk prediction models could be generated further with these radiomic features.

\section{Acknowledgements}

None.

\section{Conflict of interest}

Authors declare that there is no conflict of interest.

\section{References}

1. Lambin P, Rios Velazquez E, Leijenaar R, et al. Radiomics: extracting more information from medical images using advanced feature analysis. Eur J Cancer. 2012;48(4):441-446.

2. Kumar V, Gu Y, Basu S, et al. Radiomics: the process and the challenges. Magn Reson Imaging. 2012;30(9):1234-1248.

3. Robert J Gillies, Paul E Kinahan, et al. Radiomics: images are more than pictures, they are data. Radiology. 2016;278(2):563-s577.

4. Yip SS, Aerts HJ. Applications and limitations of radiomics. Phys Med Biol. 2016;61(13):R150-166.

5. Parekh, Vishwa Jacobs, Michael A. Radiomics: a new application from established techniques. Expert Review of Precision Medicine and Drug Development. 2016;1(2):207-226.

6. Strepen SF yip. Associations between radiologist-defined semantic and automatically computed radiomic features in non-small cell lung cancer. Scientific Reports. 2017;7:3517. 
7. Chicklore S, Goh V, Siddique. Quantifying tumour heterogeneity in 18F-FDG PET/CT imaging by texture analysis. European Journal of Nuclear Medicine and Molecular Imaging. 2012;40(1):133-140.

8. Cook Gary JR, Siddique M, Taylor Benjamin P, et al. Radiomics in PET: principles and applications. Clinical and Translational Imaging. 2014;2(3):269-276.

9. Kumar V, Gu Y, Basu S, et al. Radiomics: the process and the challenges. Magn Reson Imaging. 2012;30(9):1234-1248.

10. Haralick RM, Shanmugam K, Dinstein I. Textural features for image classification. IEEE Transactions on Systems, Man, and Cybernetics SMC. 1973;3(6):610-621.

11. Galloway, Mary M. Texture analysis using gray level run lengths Computer Graphics and Image Processing. 1975;4(2):172-179.

12. PentlandAP. Fractal-based description of natural scenes. IEEE Transactions on Pattern Analysis and Machine Intelligence PAMI. 1984;6(6):661-674.

13. Amadasun M, King R. Textural features corresponding to textural properties. IEEE Transactions on Systems, Man, and Cybernetics. 1989;19(5):1264-1274.

14. Thibault G. Advanced statistical matrices for texture characterization: application to cell classification. IEEE Transactions on Biomedical Engineering. 2014;61(3):630-637.

15. Depeursinge A, Al Kadi, Omar S, et al. Biomedical Texture Analysis: Fundamentals, Tools and Challenges; 2017.

16. Gu Y, Kumar V, Hall Lawrence O, et al. Automated delineation of lung tumors from CT images using a single click ensemble segmentation approach. Pattern Recognition. 2013;46(3):692-702.

17. Velazquez, Emmanuel Rios, Parmar C, et al. Volumetric CT-based segmentation of NSCLC using 3D-slicer. Scientific Reports. 2013;3:3529.

18. Parmar C, Leijenaar Ralph TH, Grossmann Patrick, et al. Radiomic feature clusters and prognostic signatures specific for Lung and head \& neck cancer. Scientific Reports. 2015;5:11044.

19. Tixier F, Le Rest CC, Hatt M, et al. Intratumor heterogeneity characterized by textural features on baseline 18F-FDG PET images predicts response to concomitant radio chemotherapy in esophageal cancer. J Nucl Med. 2011;52(3):369-378.

20. Hatt M, Majdoub $M$, Vallières $M$, et al. 18F-FDG PET uptake characterization through texture analysis: investigating the complementary nature of heterogeneity and functional tumor volume in a multi-cancer site patient cohort. J Nucl Med. 2015;56(1):38-44.
21. Van Rossum PS, Fried DV, Zhang L, et al. The incremental value of subjective and quantitative assessment of $18 \mathrm{~F}-\mathrm{FDG}$ PET for the prediction of pathologic complete response to preoperative chemoradiotherapy in esophageal cancer. J Nucl Med. 2016;57(5):691-700.

22. Yip SS, Coroller TP, Sanford NN, et al. Relationship between the temporal changes in positron-emission-tomography-imaging-based textural features and pathologic response and survival in esophageal cancer patients. Front Oncol. 2016;29(6):72.

23. Zhang H, Tan S, Chen W, et al. Modeling pathologic response of esophageal cancer to chemoradiation therapy using spatial-temporal 18F-FDG PET features, clinical parameters, and demographics. Int J Radiat Oncol Biol Phys. 2014;88(1):195-203.

24. Cheng NM, Fang YH, Lee LY, et al. Zone-size nonuniformity of 18 F-FDG PET regional textural features predicts survival in patients with oropharyngeal cancer. Eur J Nucl Med Mol Imaging. 2015;42(3):419-428.

25. Cook GJ, Yip C, Siddique M, et al. Are pretreatment 18F-FDG PET tumor textural features in non-small cell lung cancer associated with response and survival after chemo radiotherapy. J Nucl Med. 2013;54(1):19-26.

26. Coroller TP, Grossmann P, Hou Y, et al. CT-based radiomic signature predicts distant metastasis in lung adenocarcinoma. Radiother Oncol. 2015;114(3):345-350.

27. Vallières M, Freeman CR, Skamene SR, et al. A radiomics model from joint FDG-PET and MRI texture features for the prediction of lung metastases in soft-tissue sarcomas of the extremities. Phys Med Biol. 2015;60(14):5471-5496.

28. Brown R, Zlatescu M, Sijben A, et al. The use of magnetic resonance imaging to noninvasively detect genetic signatures in oligodendroglioma. Clinical Cancer Research. 2008;14(8):2357-2362.

29. Drabycz S, Roldán G, De Robles P, et al. An analysis of image texture, tumor location, and MGMT promoter methylation in glioblastoma using magnetic resonance imaging. Neuroimage. 2010;49(2):1398-1405.

30. Utman DA, Dunn WD, Grossmann P, et al. Somatic mutations associated with MRI-derived volumetric features in glioblastoma. Neuroradiology. 2015;57(12):1227-1237. 